\title{
An automatic recording system of the positional behavior of mice for experimental analysis
}

\author{
TAKEHISA HIRAO, HARUHIKO SHINOZAKI, NOBUHIKO FURUYA, and MICHIO ISHIKAWA \\ Department of Behavior and Physiology. Behavior Research Institute \\ University of Gunma Medical School, 3, Showa-mar'hi, Maebashi, Japan
}

\begin{abstract}
A device for automatic recording of a mouse positional behavior within a circular corridor-like cage is described. The animal's position is successfully monitored by capacitor detection and its time spent at each position measured; the results are punched on the paper tape as binary coded numbers, and the data are processed off-line by a general-purpose computer.
\end{abstract}

The positional behavior of a small animal, such as a mouse, confined in a cage with numbered unit spaces, can be represented by a sequence of addresses of unit spaces. Three measures were considered. (1) The length of a passage measured by the number of unit spaces traversed by the animal in a bout (see below). This measure was independent of cage size. (2) The frequency of passage in a given time, which depended. in part, upon the circadian thythm of activity level. (3) And the multinomial distribution of the time spent in each unit space. This could be evaluated and expressed as "entropy," showing the nonrandomness of the animal's preference for the spaces in the cage.

Hirao and Utena (1962) described an apparatus (the "cloister") by which the position of a mouse could be monitored by capacitor detection and recorded automatically. The device consisted of a circular corridor divided into unit spaces; each space was a little larger than the animal and was coded by number (Figure 1). Hirao, Shinozaki, Furuya, and Ishikawa (1973) improved the system so that the data were processed and analyzed with a general-purpose computer. Lewin (1938) described a similar analytical method. The Lewin method was designed to study the "direction of psychological forces" by tracking the subject's movements projected onto a "hodological space" divided into unit spaces. The method, however, was not tested experimentally.

The purpose of this paper is to describe a useful method that has undergone a series of tests to eliminate technical difficulties originally associated with the system.

\section{The Apparatus and Circuitry}

The apparatus was designed for a mouse. The mouse was placed in a circular corridor, where it

The authors would like to thank Professor M. Cullen, Department of Experimental Psychology, University of Oxford, for his critical reading of the manuscript. $N$. Furuya's present address is Department of Physiology, Nippon Medical School. H. Shinozaki and $M$. Ishikawa are presently in the Research Laboratory, Pharmaceutical Division, Nippon Kayaku Co., Ltd.

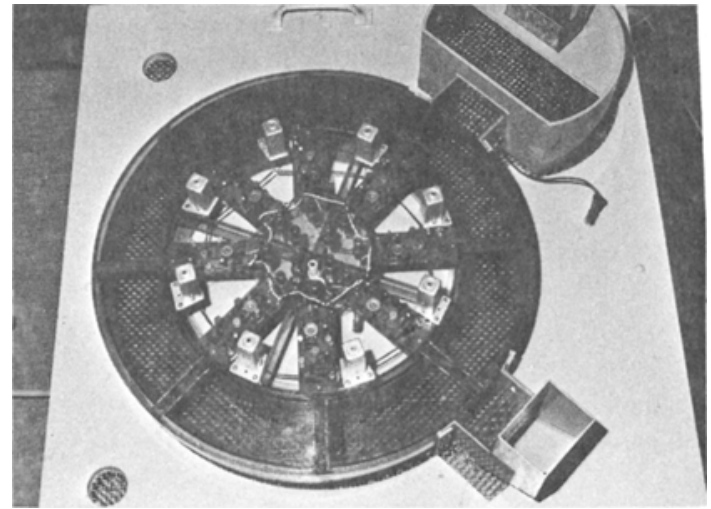

Figure 1. Cloister from the top.

could run freely or stay in place. Its presence in any segment of the corridor was monitored by a capacitor-detection technique. The roof and wall of the corridor were made of transparent Plexiglas. The vertical section was rectangular (height, $7.5 \mathrm{~cm}$, and width, $10 \mathrm{~cm}$ ), and the corridor-like cage was divided into numerical unit spaces determining the size of the cage. Metal meshes of $14 \times 2 \mathrm{~cm}^{2}$ were placed on the outer surface of the roof $15 \mathrm{~cm}$ apart; these were used as reference electrodes. The floor was of rough metal mesh through which waste material could fall. The floor served as the ground for the electrical circuitry. Thus, each space between the ceiling electrode and the floor was the unit space with a monitor system (Figure 1). The volume of unit space in which a mouse could be measured was $7.5 \times 10 \times 15 \mathrm{~cm}^{3}$.

Mice were housed in this cage for several months without external disturbance except for feeding and watering.

A block diagram of the monitor system is illustrated in Figure 2. A capacitor between the reference electrode on the ceiling and the floor forms an LC resonator with the input coil; it is tuned at $1.8 \mathrm{MHz}$. When a mouse enters a unit space from a neighboring 


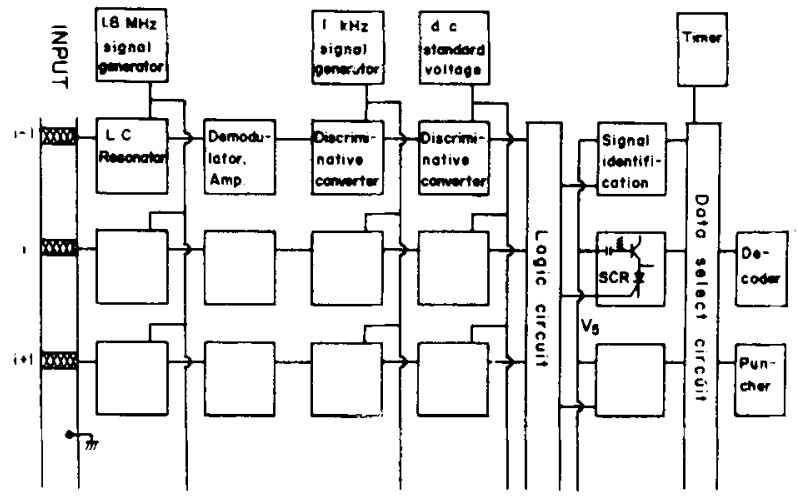

Figure 2. Block diagram of the detect system. Details in the text.

space, the resonator is detuned and the signal voltage decreases. When the mouse leaves this space, the signal voltage returns to the original high level. The signal wave is demodulated, amplitied, and converted to a standard $1-\mathrm{kHz}$ wave by the converter-amplifier to increase the $S / N$ ratio. These signals, coming from the inputs of all the unit spaces, are led to a logic circuit where only an on-state signal of the mouse's presence in a unit space is allowed to occur. The off-state signal is made when the on-state signal of the neighboring spaces occurs. Such on and off signals. together with the address code of the unit space. are encoded in the data select circuit. Whenever, the mouse changes position, the information is punched onto 8-bit paper tape as a binary coded number. All punched data are processed by a general-purpose computer OKITAC-5090.

\section{Capacitor-Detector Circuit}

As shown in Figure 3, the 1.8- $\mathrm{MHz}$ signal wave, amplitude-modulated at $1 \mathrm{kHz}$, is supplied from an external oscillator to the input coil. T, of each LC resonator. The resonance is adjusted with a variable capacitor. $C_{1}$, and the resonance voltage is demodulated by a diode. $D_{1}$, amplified by an operational amplitier. $A_{1}$, rectified by a diode, $D_{2}$, then changed to direct current (dc) voltage through an LC filter. This system provides high stability and sufficient sensitivity to detect the position of the mouse and adjustments are unnecessary. Of course. the higher the frequency and amplitude of the carrier wave, the more remarkable is the mutual interference, because of the paralleled input LC resonators nearby. Trial and error indicates that the carrier wave should be fixed at a frequency of $1.8 \mathrm{MHz}$ and at a voltage of $180 \mathrm{mV}$. The amplitude modulation (by $1-\mathrm{kHz}$ sine wave) is convenient to obtain the high gain and stable amplifying.

The de voltage signal after an LC filter is injected to the noninverting input of the operational amplifier, $A_{2}$. and the $1-k H z$ reference sine wave $\left(V_{2}\right)$ is fed to the inverting input of $\mathrm{A}_{2}$ through an FET follower, $\mathrm{Q}_{3}$. The $\mathrm{V}_{2}$ amplitude is adjusted by a variable

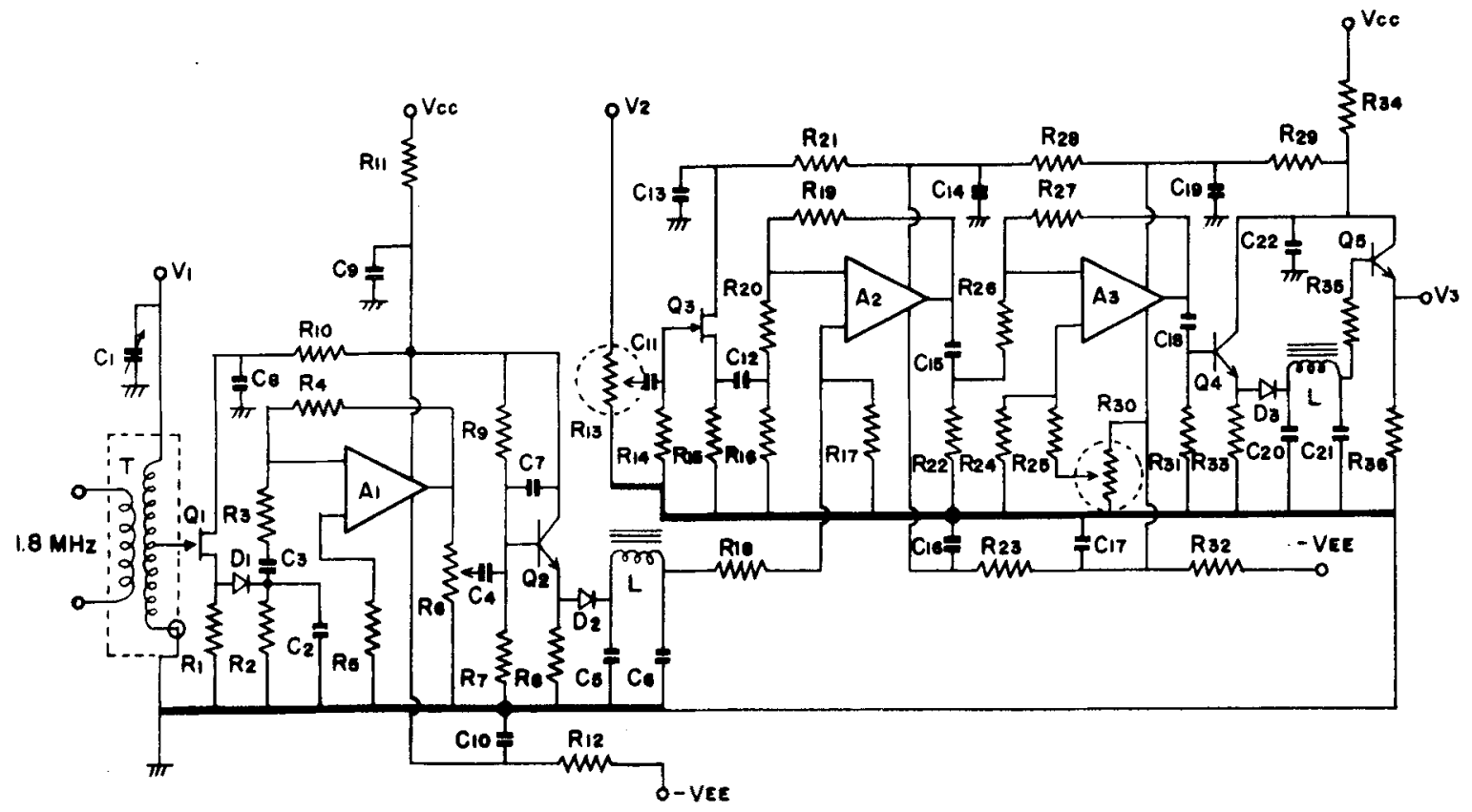

Figure 3. Capacitor-detection and amplification. $\mathbf{R}_{1}, \mathbf{R}_{7}, \mathbf{R}_{10}, \mathbf{R}_{21}, \mathbf{R}_{31}, \mathbf{R}_{35}: 10 \mathrm{~K} \Omega ; \mathbf{R}_{2}, \mathbf{R}_{9}: 100 \mathrm{~K} \Omega ; \mathbf{R}_{3}, \mathbf{R}_{5}: 2 \mathrm{~K} \Omega ; \mathbf{R}_{4}, \mathbf{R}_{14}$,

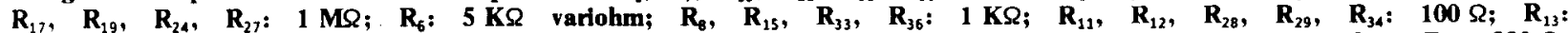
$500 \mathrm{~K} \Omega$ variohm; $R_{16}, R_{22}: 5 \mathrm{~K} \Omega ; R_{10}, R_{20}: 20 \mathrm{~K} \Omega ; \mathbf{R}_{23}: 220 \Omega ; \mathbf{R}_{25}, \mathbf{R}_{26}: 50 \mathrm{~K} \Omega ; \mathbf{R}_{30}: 20 \mathrm{~K} \Omega$ variohm; $\mathbf{R}_{32}: 330 \Omega$; $\mathrm{C}_{1}: 15 \mathrm{pF}$ variable capacitor; $\mathrm{C}_{2}, \mathrm{C}_{6}: 0.01 \mu \mathrm{F} ; \mathrm{C}_{3}, \mathrm{C}_{4}: 2.2 \mu \mathrm{F} ; \mathrm{C}_{3}, \mathrm{C}_{11}, \mathrm{C}_{20}, \mathrm{C}_{21}: 0.1 \mu \mathrm{F} ; \mathrm{C}_{7}: 100 \mathrm{pF} ; \mathrm{C}_{8}, \mathrm{C}_{13}, \mathrm{C}_{14}$, $\mathrm{C}_{16}, \quad \mathrm{C}_{17}, \quad \mathrm{C}_{19}, \quad \mathrm{C}_{22}: 10 \mu \mathrm{F} ; \mathrm{C}_{9}, \mathrm{C}_{10}: 33 \mu \mathrm{F} ; \mathrm{C}_{12}, \mathrm{C}_{15}: 4.7 \mu \mathrm{F} ; \mathrm{C}_{18}: 2.2 \mu \mathrm{F} ; \mathrm{T}:$ Trio T-21; $\mathbf{Q}_{1}, \mathrm{Q}_{3}: \mathrm{MK}_{10}$; $\mathbf{Q}_{2}$, $Q_{4}, Q_{5}: 2 S C 372 G-0 ; D_{1}, D_{2}, D_{3}: 1 S 1555 ; A_{1}, A_{2}, A_{3}: S N 72741 ; L: S T \cdot 30 ; V_{1}:$ input for the ceiling electrode; $V_{2}:$ input for the reference 1.KHz sine wave; $V_{3}$ : output to the logic identification circuit; $V_{\text {ce: }}+15 V_{\text {; }}-V_{E E}:-15 V$. 
resistance, $R_{13}$ (encircled by a broken line), to make the $A_{2}$ output voltage smallest when there is no mouse in the unit space. Finally, an operational amplifier, $A_{3}$ eliminates the surplus signal from $\mathrm{A}_{2}$. Potentiometer $\mathrm{R}_{30}$ (encircled by a broken line) provides a standard negative de bias to the noninverting input of $\mathrm{A}_{3}$ to keep the output voltage at zero level during absence of a mouse. The output signal of $\mathrm{A}_{3}$ is rectified by a diode, $D_{3}$, and is changed again to de voltage, $V_{3}$.

\section{Identification of the Signal Indicating Mouse Position}

A total of $\mathrm{V}_{3}$ signals from all unit spaces is lead to the logic circuit shown in Figure 4. so that the signals of two neighboring detectors are not activated simultaneously even if the mouse is in a boundary between the unit spaces and occupying neighboring two unit spaces. An inverter, $I_{1}$, and a positive-AND gate, $G_{3}$, are provided for each unit space. Odd and even groups of the unit spaces have positive-NAND gate, $G_{1}$. respectively, and both groups possess a common NAND gate, $G_{2}$. In this circuitry, more than two outputs of the positive-AND gate, $G_{3}\left(V_{4}\right)$, cannot be logical "1." Each output of $\mathrm{V}_{4}$ is coupled to the gate of respective silicon-controlled rectitier (SCR) shown in Figure 5. Once the gate level is increased

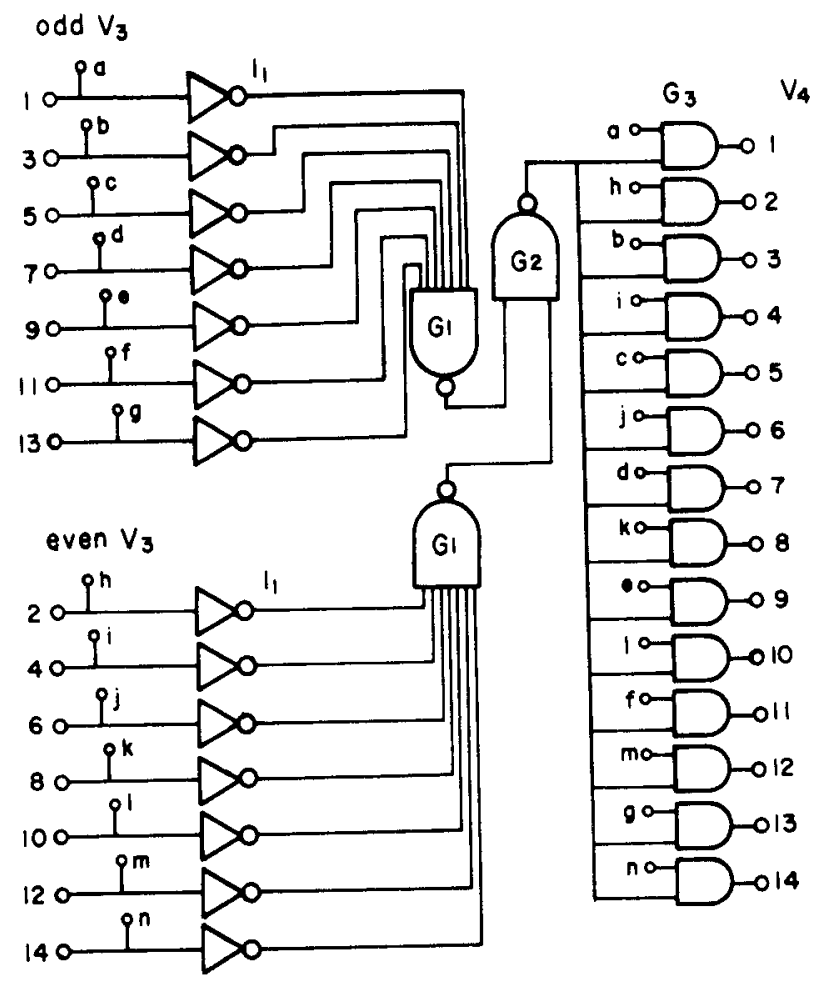

Figure 4. A logic circuit enabling the on signal only at one unit space for the presence of the mouse. $I_{1}$ : inverter; $G_{1}, G_{2}$ : positive-NAND gate; $G_{3}$ : positive-AND gate; $V_{3}$ : input; $V_{4}$ : output. The colateral terminal marked alphabetically in each input $\left(V_{3}\right)$ is connected with the relevant output of positive-AND gate $\left(V_{4}\right)$, which is indicated by the same alphabet.

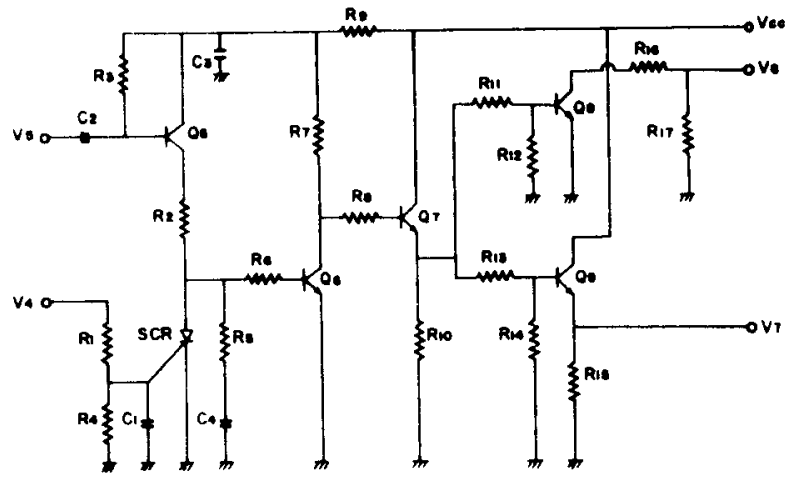

Figure 5. Composing circuit of the presence signal at the unit space. $\mathbf{R}_{1}, \mathbf{R}_{4}: 1 \mathrm{~K} \Omega ; \mathbf{R}_{2}: 200 \Omega ; \mathbf{R}_{3}: 200 \mathrm{~K} \Omega ; \mathbf{R}_{5}: 20 \Omega ; \mathbf{R}_{6}, \mathbf{R}_{8}, \mathbf{R}_{12}$, $R_{14}: 10 \mathrm{~K} \Omega ; R_{7}: 3 \mathrm{~K} \Omega ; R_{9}: 100 \Omega ; R_{10}, R_{15}: 2 \mathrm{~K} \Omega ; R_{11}, R_{13}: 5 \mathrm{~K} \Omega$; $R_{16}: 20 \Omega 1 / 2 W ; R_{17}: 500 \Omega 1 / 2 W ; C_{1}, C_{2}, C_{4}: 0.1 \mu F ; C_{3}: 10 \mu F ; Q_{5}$ ... $Q_{9}$ : 2SC372G-0; SCR: SFOR2B-41; $V_{4}$ : input for the signal current; $V_{5}$ : input for the controlling transistor (all are connected together); $V_{6}$ : output to the pilot lamp; $V_{7}$ : output to the data selector; $V_{\text {ec: }}+15 \mathrm{~V}$.

over a threshold value (about $0.6 \mathrm{~V}$ ) by a mouse appearing in the unit space, the corresponding SCR continues firing until the controlling transistor, $Q_{5}$, works. Thus, the reduction of the anodal voltage represents an "on" signal (the mouse is at the unit space) and is conducted to the bases of Transistors $Q_{5}$ of all the other unit spaces by a common line, $V_{5}$, to turn off the firing SCRs of the other unit spaces. A series of transistors, $Q_{6}, Q_{7}, Q_{8}$, and $Q_{9}$, serve as amplitiers or buffers developing an appropriate voltage level of the two outputs, one for driving the pilot lamp. $V_{6}$ of visual monitor, for the mouse position, and the other for the data input, $V_{7}$, of the

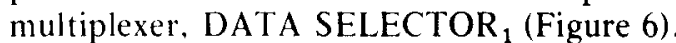

\section{Encoding System of the Address Number and the Staying Time (Figure 6)}

Data signals are reduced to a set of symbols by applying the appropriate binary-coded address of the data selector inputs (A, B, C, D) taken from the

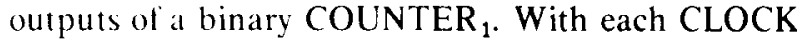
PULSE whose frequency is $2 \mathrm{kHz}$, the COUNTER, switches to the next state, causing the DATA SELECTOR 1 to select in sequence the information source connected to its inputs, $V_{7}$. When data are selected. the output of the DATA SELECTOR changes from a logical " 0 " to a logical " 1 ." triggering a monostable multivibrator $\left(\mathrm{MM}_{1}\right)$ to produce a pulse as a transfer signal, which makes the $\mathrm{LATCH}_{1}$ function and COUNTER ${ }_{1}$ clear and reset through another multivibrator $\left(\mathrm{MM}_{2}\right)$. Therefore, the output of the $\mathrm{LATCH}_{1}$ represents the specific coded address number of the unit space where the mouse is present. The change of mouse position produces a change in the output code of the $\mathrm{LATCH}_{1}$, inducing a pulse through multivibrators $\left(\mathrm{MM}_{3}\right)$ and $\mathrm{OR}$ gate $(\mathrm{G})$. This pulse is used as a clutch signal for the tape punch, enabling the $\mathrm{LATCH}_{3}$ function and reseting the 


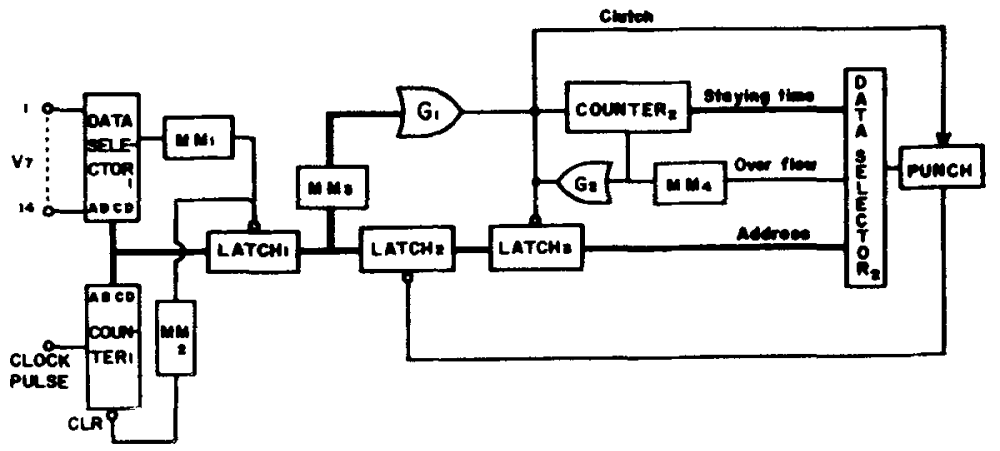

Figure 6. Block diagram of the data select circuit for the cloister apparatus $(14$ unit spaces). $V_{7}$ : Data input coming from $V$, in Figure 5. MM: Monostable multivibrator circuit for several microseconds.

COUNTER ${ }_{2}$, which is used for measuring the time spent by the mouse in a unit space.

On the paper tape, the address is punched out on the first line and the staying time (14 bits) on the second and third lines. When the staying time of a mouse is over $2^{14} \times 10^{-1} \mathrm{sec}(27 \mathrm{~min}$ and $18.4 \mathrm{sec})$, the clutch signal is produced and the overflow mark is punched on the tirst line, the blank space on the next two lines. When the punch finishes recording the output code of the $\mathrm{LATCH}_{3}$. a pulse is produced to enable the $\mathrm{LATCH}_{2}$ function. Therefore, the output code of the $\mathrm{LATCH}_{2}$ changes with $240 \mathrm{msec}$ delay for recording. and $\mathrm{LATCH}_{3}$ represents the address of the unit space where the mouse was previously.

When the transfer signal is developed during the operation of the tape punch, the clutch signal only resets the COUNTER ${ }_{2}$ for the next staying time; this is designed not to trigger the tape punch.

\section{Discussion}

We designed the cage with the unit spaces so that any particular shape or environment would be avoided as much as possible. In the circular corridor-like cage, the animal is allowed an equal possibility of going forward or backward.

The unit space is approximately the minimum volume which a small animal can occupy under laboratory conditions (Worden, 1957), and in which a nouse does not exhibit signs of gastric stress-ulcer (Bontils \& Lambling, 1963). The maximum volume where a mouse restrained by one foot can move is a half sphere with a diameter length of a mouse's body, that is. about $10 \mathrm{~cm}$. Considering the experimental technique, we eventually chose the unit space to be 7.5 × $10 \times 15 \mathrm{~cm}^{3}$. Therefore, the "minor" movement revealed within the dimension is ignored. The volume of the unit space chosen by Hirao and Utena (1962, 1965). $7 \times 10 \times 10 \mathrm{~cm}^{3}$. resulted in technical problems; for example. tail movements often disturbed position recording.

It is essential that the position of a mouse be determined one unit at a time. Sometimes this is ditficult when two neighboring sensors are triggered at the same time (e.g.. by the body and the tail). It this occurs. the recording rule of preference for the tirst measured position $i$ assured by using the
AND-NAND logic circuitry shown in Figure 4 and by the SCR characteristic shown in Figure 5. The "on" signal of the mouse's presence occurs only at a unit space by the former circuitry; the "off" signal is produced by the "on" signal of the neighboring unit space. This is useful in avoiding the ambiguous animal signal at the boundary and considerably increases the reliability of the digital recording system.

Before this apparatus was developed. a number of other methods were tried, but all proved unsalisfactory. including the photo-transistor method and special sensor elements such as strain gauges, pressure sensors, touch diodes, etc. Most required special devices inside the apparatus that disturbed animal activity or allowed waste material to interfere with detection.

The capacitor-detection method does not require complicated mechanisms inside the cage and the base plate with the input circuit can easily be removed for cleaning. Other techniques using resonance circuitry ffor instance. the oscillator detector of frequency in the input resonator) are available also. However, such devices are unsatisfactory because of mutual electrical interference among the respective circuits. and difficulties of tuning. or other adjustments.

In our research, the system was required to work continuously with high stability and suitable sensitivity. The troubles we encountered were due mainly to the temperature-depending characteristic of the diodes and to the low $\mathrm{S} / \mathrm{N}$ ratio. The demodulated signal in the input circuitry fluctuated with temperature change, but this was successfully avoided by using a silicon diode as a polarity-depending resistance of an emitter follower in the integration circuit. and the circuitry functioned stably in good temperature characteristic. When the input resonator is detuned by the presence of a mouse, the amplitude of the carrier wave in the tap of the input coil decreases by about $30 \%$; therefore. more than $70 \%$ of the amplitude of the carrier wave is still large and is a source of constant noise for the signal. The transformations and rectifications of the signal wave using the operational amplifiers are an advantage in increasing the $S / N$ ratio. Special attention must be paid to the current leakage in the input circuit due to 
the increment of humidity, particularly through the reference electrodes on the roof of the cloister.

This apparatus works with find stability at a temperature of $5^{\circ}-35^{\circ} \mathrm{C}$ and a humidity of $20^{\sigma_{0}}-90^{\circ}$ without adjustment for at least a month.

In order to record economically the data obtained during the experiment (about 2 months), our tape punch was driven by the transfer signal produced by the mouses changing position. However, the speed of mouse movement was influenced by the condition of the floor mesh (Hirao \& Utena. 1965: Hirao et al.. 1973). This was fixed so that the maximum running speed of a mouse was $1 \mathrm{~m}$ sec: that is. a mouse could pass through a unit space in $150 \mathrm{msec}$. Therefore, any positional change that lasted less than 150 msec or which occurred during the recording period of $240 \mathrm{msec}$. was not punched on the tape; these were supplemented by the sorfware which measured the duration of a visit to the nearest 100 msec when the data was processed by the computer. Due to the repeated operation of the punch and the rounding errors of timing. there were slight discrepancies in total time elapsed compared with time recorded by the apparatus. However, these can be ignored since they comprise less than 3 min in a day $(=0.2 \%)$.

Several calculations can be performed on the raw data. We found four indices to be useful (Hirao \& L'tena. 1965: Hirao et al.. 1973): (1) Length of "passage" (measured by the number of unit spaces traversed by a mouse until it stopped in a unit space for more than $1.5 \mathrm{sec}$. The length followed the lan of geometrical distribution probability, and the mean value was constant for the adult mice of several strains. The choice of criterion time is discussed by Hirao et al. (1973). (2) Frequency of passage in a given time was measured and showed a circadian rhvthm with particular characteristics identitiable for each strain. The product of the mean value of (1) and $(2)$ is equal to the locomotory activity level in a given time. (3) The spatial distribution of the animal location is represented by the "entropy" $H$, as follows:

$$
H_{v, n}=\sum_{i=1}^{n} p_{v, i} \log _{2} p_{v i, i}
$$

where

$\mathrm{p}_{\mathrm{v} . \mathrm{i}}=$

(total number of visits to the unit space numbered i) (total number of visits to all unit spaces)

and

$$
H_{s . \mathrm{ll}}=-\sum_{i=1}^{n} p_{s . i} \log _{2} p_{s, i}
$$

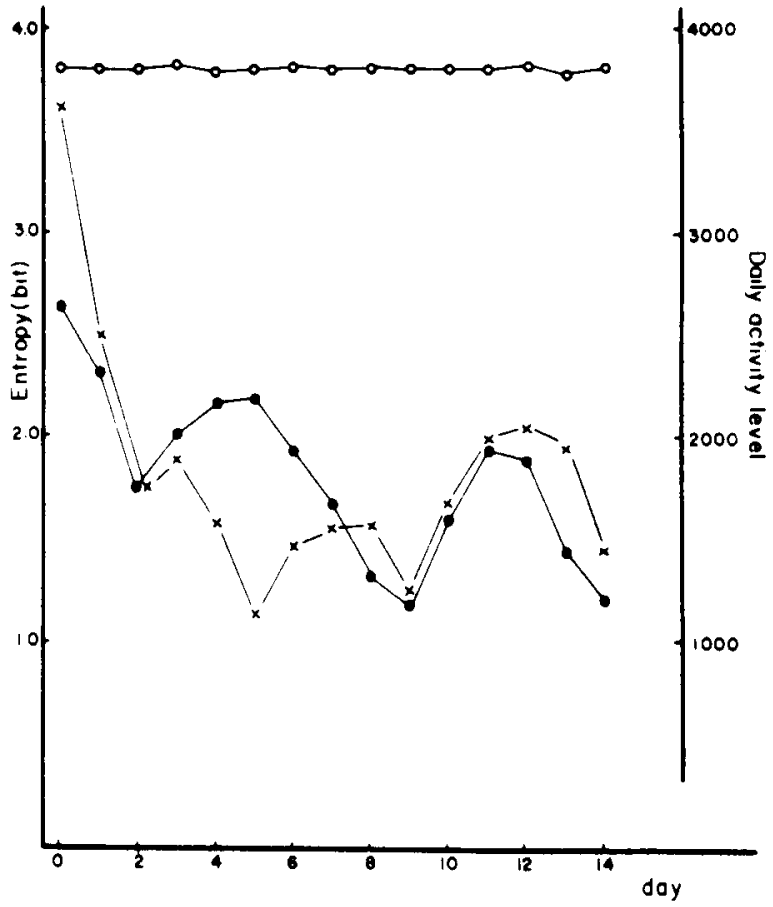

Figure 7. Changes of entropy and daily activity level along the habituation process in the cloister. $H_{v}$ (open circles) obtained from the visiting frequency, and $\mathrm{H}_{\mathrm{s}}$ (closed circles) from the staying time. Daily activity level (xs) is also shown.

where

$P_{s, i}=$

(total time spent in the unit space numbered i) (total time spent in all unit spaces)

The suftix $n$ denotes the total number of unit spaces. i.e.. the cage size, and $i$ denotes the particular unit space. Thus. $\mathrm{H}_{y, n}$ describes the nonrandomness of the mouse entering a unit space, and $\mathrm{H}_{\mathrm{s} . \mathrm{n}}$ describes the nomrandomness of staying. In order to compare the positional behavior of the animal in various circumstances. and because the entropy depends on the calge-size. (4) relative entropy can be calculated for $\mathrm{H}_{1, n}$ and $\mathrm{H}_{\mathrm{S} . n}$ as a fraction of the maximum value of entrops.

Figure shows an example of the change of the entrop! thus calculated during the habituation process of ddN strain in the cloister. In this tigure, the abscissa represents the day elapsed since introduction. and the ordinate the entropy $\mathrm{H}_{S}$ and $\mathrm{H}_{\mathrm{V}}$ in $24 \mathrm{~h}$. The former entrops decreased gradually for the first 3 dars and then attained a stationary level. while the latter entropy kept the maximum level throughout the experiment.

\section{REFERENCES}

Bonfils. S.. A Lambling. A. Psychological factors and psycho. pharmacological ations in the restraint-induced gastric ulcer. 
In S. C. Skoryna (Ed.). Pathophysiology of peptic ulcer. Montreal: McGill University Press. 1963. Pp. 153-171.

Hirao, T., Shinozaki, H., Furuya, N., \& Ishikawa, M. A new behavior measuring system: Cloister method (in Japanese with English summary). Journal of the Physiological Society of Japall, 1973, 35, 10-18.

Hirao, T., \& Utena, H. New instrument to measure the directed movement of a mouse (in Japanese). Protein. Nucleic Acid and Enzymo'. 1962. 7. 789.

Hirao, T., \& UTEnA. H. Methodological considerations on the measurement of behavior of an animal: Cloister method (in Japanese with English summary). Psychiatry and Neurology of Japan, 1965, 67, 1005-1023.

LEWIN. K. The conception of representation and the measurement of Psychological forces. Durham: Duke University Press. 1938.

WORDEN, A. The UFA $W$ hand book on the care and management of laboratory animals. London: The University Federation for Ánimal Welfare, 1957.

(Received for publication October 24, 1974; revision received December 21,1974 .) 